\title{
Simplifying Protocols on MRI in Pediatric Oncology-Personalized Focused Assessment Rapid Magnetic Resonance Imaging on pediatrics
}

\author{
Jose Luiz de Oliveira Schiavon · Jeannie Romani • \\ Henrique Manoel Lederman
}

Published online: 18 June 2014

(C) Springer Science+Business Media New York 2014

\begin{abstract}
The use of axial imaging studies has been increasing fast all over the world regardless the efforts to reduce radiation exposure on computed tomography (CT) scans, which magnetic resonance imaging (MRI) can play a role over most indications. Along with the radiation of CT, and the increased risk of sedation when needed, advances in MRI technique are required to reduce scan time, depending on the indication of the examination, allowing more patients to be scanned hourly and reducing the use of CT scanner and sedation. With limitations on mind, personalized studies should be carried to children according to its indication. This article will describe one institution experience of personalized focused assessment rapid MRI on pediatrics, where most pediatric patients can be followed up using MRI-personalized protocols, avoiding the use of CT and allowing more patients to be scanned hourly, that is useful on locations where MR scanners still of low availability, keeping in mind that a good interaction
\end{abstract}

This article is part of Topical Collection on MRI for Pediatric Oncology.

J. L. de Oliveira Schiavon

UNIFESP - Universidade Federal de Sao Paulo, Sao Paulo,

Brazil

e-mail: schiavon00@gmail.com

\section{J. Romani}

IOP-UNIFESP/GRAACC - Instituto de Oncologia Pediatrica da Universidade Federal de Sao Paulo/Hospital do GRAACC, Sao Paulo, Brazil

e-mail: jeannieromani@hotmail.com

H. M. Lederman ( $₫)$

Departamento de Diagnóstico por Imagem, Universidade Federal de Sao Paulo DDI - UNIFESP, Sao Paulo, Brazil

e-mail: lederman@unifesp.br between the radiologists and radiologic technician is needed to determine the ideal parameters for reading.

Keywords Pediatric - Oncology · Protocols · Fast · Simplified · Magnetic Resonance Imaging

\section{Introduction}

The use of axial imaging studies has been increasing fast all over the world, regardless the efforts to reduce radiation exposure on computed tomography (CT) scans, which magnetic resonance imaging (MRI) can play a role over most indications, MRI scanners are not as widely available as CT, especially on developing countries, and when present, the standard study time of the majority of scanners allows only a limited number of patients to be examined hourly and may require sedation on some patients.

Pediatric population, who also often have, along with the radiation of $\mathrm{CT}$, the increased risk of sedation when needed, advances in MRI technique are required to reduce scan time, depending on the indication of the examination, allowing more patients to be scanned hourly and reducing the use of CT scanner and sedation. Unfortunately, MRI scanners are extreme sensible to motion artifacts and are also fear and anxiety generator for the majority of young children [1]. According to these aspects, Dean et al. [2] on 2013 suggested that mcDESPOT (multicomponent driven equilibrium single pulse observation of $\mathrm{T} 1$ and $\mathrm{T} 2$ ) protocols could reduce scan time up to 18-25 min, depending on the age, for brain MRI on 220 children, permitting them to be scanned during non-sedated sleep on $97 \%$. Oslen 2013 [3] also contributed to pediatric MRI with protocols adjustments, to reduce motion artifacts and the poor signalto-noise ratio, using coils with element sizes appropriate 
for the anatomy of children, using contrast, and paying attention to the children preparation such as the flip-side positioning.

Table 1 Comparison of routine and fast parameters of brain and face protocols that helps on time reduction, these adjustments can be done with care and keeping in mind that a good interaction between the radiologists and radiologic technologists is needed to determine the ideal parameters for reading

\begin{tabular}{llll}
\hline Fast flair-brain & Routine flair-brain & Fast T2-face & Routine T2-face \\
\hline RT 11000 & RT 11000 & RT 6716 & RT2065 \\
ET 140 & ET 2800 & ET 120 & ET 120 \\
IT 2800 & IT 140 & TSE 16 & TSE 20 \\
Matrix 320 & Matrix 704 & Matrix 576 & Matrix 384 \\
Nex 2 & Nex 2 & Nex 3 & Nex 4 \\
Sense 1,5 & Sense no & Sense 1,6 & Sense no \\
\hline
\end{tabular}

Table 2 Comparison time between a complete and a fast protocol parameters for the Brain studies

\begin{tabular}{lll}
\hline & Brain-complete & Brain-fast \\
\hline Axial flair & $3: 18$ & - \\
Axial T2 & $1: 21$ & - \\
Axial T1 & $1: 40$ & $1: 28$ \\
Axial T1 post contrast & - & $1: 28$ \\
Axial SWI & $2: 49$ & - \\
Axial capilarity & $1: 07$ & - \\
Axial difusion & $0: 46$ & $0: 46$ \\
Coronal flair & - & $1: 50$ \\
Coronal T2 & $1: 18$ & - \\
Axial perfusion & $1: 08$ & - \\
Sagital T1 & - & $1: 46$ \\
Sagital 3D T1 & $5: 34$ & $5: 34$ \\
Spectroscopy & $3: 16$ & - \\
Spectroscopy & $3: 16$ & - \\
Total time & Aprox. 26 min & Aprox. 13 min \\
\hline
\end{tabular}

To ensure quality and the right use of fast MRI protocols, Rozovsky et al. 2012 [4••] studied the limitations of these protocols using only FIESTA (Fast Imaging Employing sTeady State Acquisition) sequence, that study missed findings on $14 \%$ of cases, all related to blood brain events such as venous sinus thrombosis, subdural hematoma, extraaxial collections, and differentiation of blood products.

Knowing the importance of rapid MRI studies, to improve its availability and reduce possible alternative CT with radiation exposure and sedation, with limitations on mind, personalized studies should be carried to children according to its indication as described by Leite et al. 2011 [5•] and Ashley et al. 2005 [6] who recommended specific protocols to reduce the use of CT on both pediatric sinus disease and brain diseases such as hydrocephalus.

In this article we will describe one institution experience of personalized focused assessment rapid MRI on pediatrics.

\section{Simplifying Protocols}

Adjustments on MRI parameters can lead to a time reduction on most sequences, these sequences can be

Table 3 Comparison time between a Complete and Fast protocol parameters for paranasal sinus

\begin{tabular}{lll}
\hline & Sinus-complete & Sinus-fast \\
\hline Axial flair-brain & - & $1: 50$ \\
Axial T2 & $1: 46$ & - \\
Axial T1 & $2: 10$ & $2: 30$ \\
Axial T1 post contrast & $2: 25$ & - \\
Coronal T1 Post contrast & $4: 42$ & - \\
Axial difusion-brain & - & $0: 48$ \\
Coronal T1 & $3: 07$ & - \\
Coronal T2 & $3: 05$ & $2: 14$ \\
Sagital T1 & $2: 43$ & - \\
Total time & Aprox. 20 min & Aprox. 7.5 min \\
\hline
\end{tabular}

Fig. 1 Optimized paranasal sinuses and brain MRI scan, usually searching for infection, takes only 10 min including axial FLAIR, T1, and diffusion of the brain and paranasal coronal T2. This protocol almost always eliminated the use of CT for paranasal sinuses infection

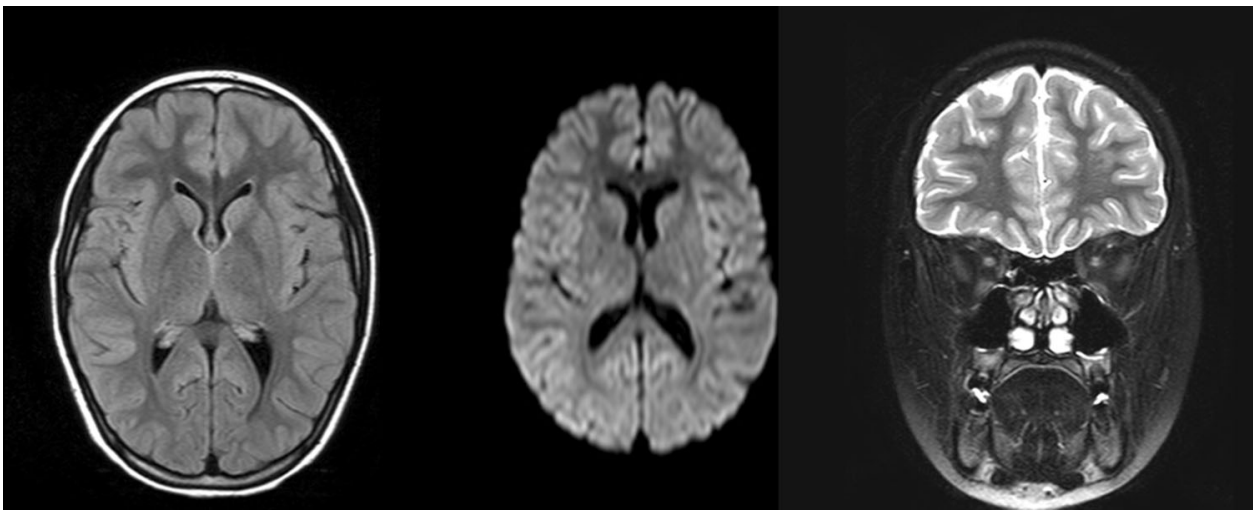


personalized according to the radiologist team reading comfort aiming not to create poor quality images. Basically, Turbo factor, Nex, Matrix, and $\operatorname{SENSE}^{\mathrm{TM}}$ (K space filling accelerator, available at Philips ${ }^{\circledR}$ SENSE $^{\mathrm{TM}}$ Coils) adjustments help on these time reductions (Table 1).

Usually, the admission study for most tumors, especially brain, includes a complete routine scan taking up to $26 \mathrm{~min}$, if not done before in any other radiology facility. After the initial evaluation, all other studies are personalized according to the referring physician indication leading to a 13-min reduction on almost all cases (Table 2).

Table 4 Comparison time between a Complete and Fast protocol parameters for brain and eyes scan

\begin{tabular}{lll}
\hline & Brain/eye complete & Brain/eye fast \\
\hline Axial flair-brain & $3: 18$ & $3: 18$ \\
Coronal T2-eye & $2: 13$ & $2: 13$ \\
Axial T2-eye & $2: 19$ & $2: 19$ \\
Axial T1-eye & $1: 57$ & $1: 57$ \\
Axial FFE-eye & $2: 06$ & $2: 06$ \\
Sagital T2 fatsat-eye R & $2: 12$ & - \\
Sagital T2 fatsat-eye L & $2: 12$ & - \\
Coronal stir-neck & $2: 13$ & - \\
Axial T2 fatsat-neck & $1: 57$ & - \\
Axial thrive GD-eye & $1: 26$ & - \\
Axial T1 GD-eye & $2: 21$ & $2: 21$ \\
Sagital T1 3D GD-brain & $5: 34$ & $5: 34$ \\
Sagital T1 GD-spine & $7: 00$ & \\
Total time & Aprox. 37 min & Aprox. 20 min \\
\hline
\end{tabular}

Optimized paranasal sinuses and brain MRI scan, usually searching for infection, takes only $10 \mathrm{~min}$ including axial FLAIR, T1, and diffusion of the brain and paranasal coronal T2 (Fig 1). This reduction decreases the scan time on over $50 \%$ when compared to the usual 22-min scan (Table 3) and should lead CT to a very limited indication aiming the reduce of radiation on children.

When retinoblastoma is suspected, an initial complete brain and eye scan is performed taking $37 \mathrm{~min}$, after the diagnostic confirmation, following scans are performed using control fast protocols that take about $20 \mathrm{~min}$, as described on Table 4 (Fig 2).

Total body studies are usually carried for lymphoma or other high cellularity and small cells tumors evaluation, search for metastasis, or over palliative care patients, detecting lesions that can cause pain, or in the search for symptoms of unknown cause.

Whole body MRI (WBMRI) is usually performed on coronal plane with Short Tau Inversion Recovery (STIR) sequence and Diffusion Weighted Images with Background Suppression (DWIBS) Maximum Intensity Projection (MIP) reconstruction. This study protocol allows radiologists to combine a very sensitive STIR sequence with a water restriction DWIBS series that can infer the cellularity barrier to water diffusion, helping determinate whether any STIR abnormal signal is correlated with high cellularity or not (Fig 3). The total study time takes about $37 \mathrm{~min}$ covering the total body, and its interpretation should be fast regardless the large amount of images, using a binary normal/abnormal signal, where only abnormal areas require further evaluation and comparison to prior or other studies.
Fig. 2 Optimized brain and eye scan for retinoblastoma example that takes about $20 \mathrm{~min}$

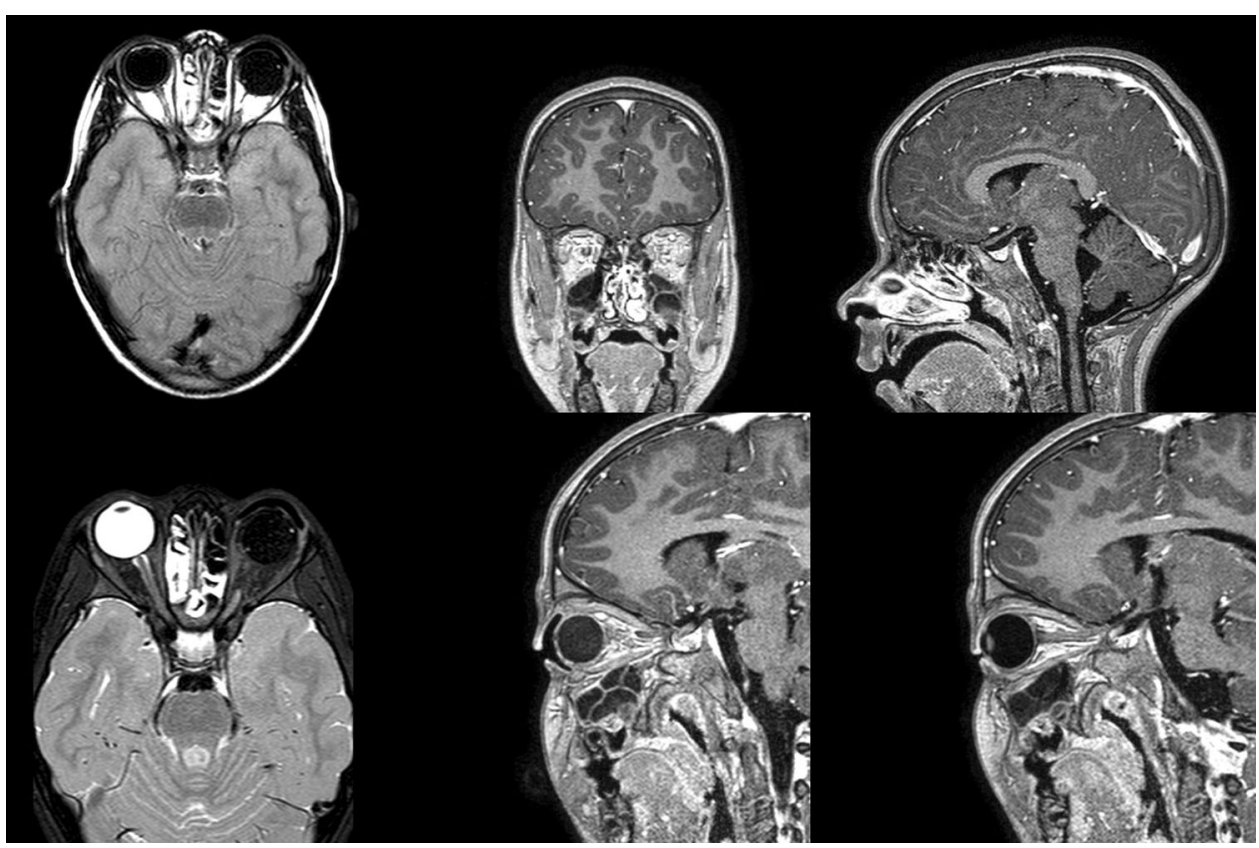


Fig. 3 Whole body MRI with Diffusion for Lymphoma follow-up showing the loss of signal of the neck on right images compared to the left ones, for both STIR and DWI

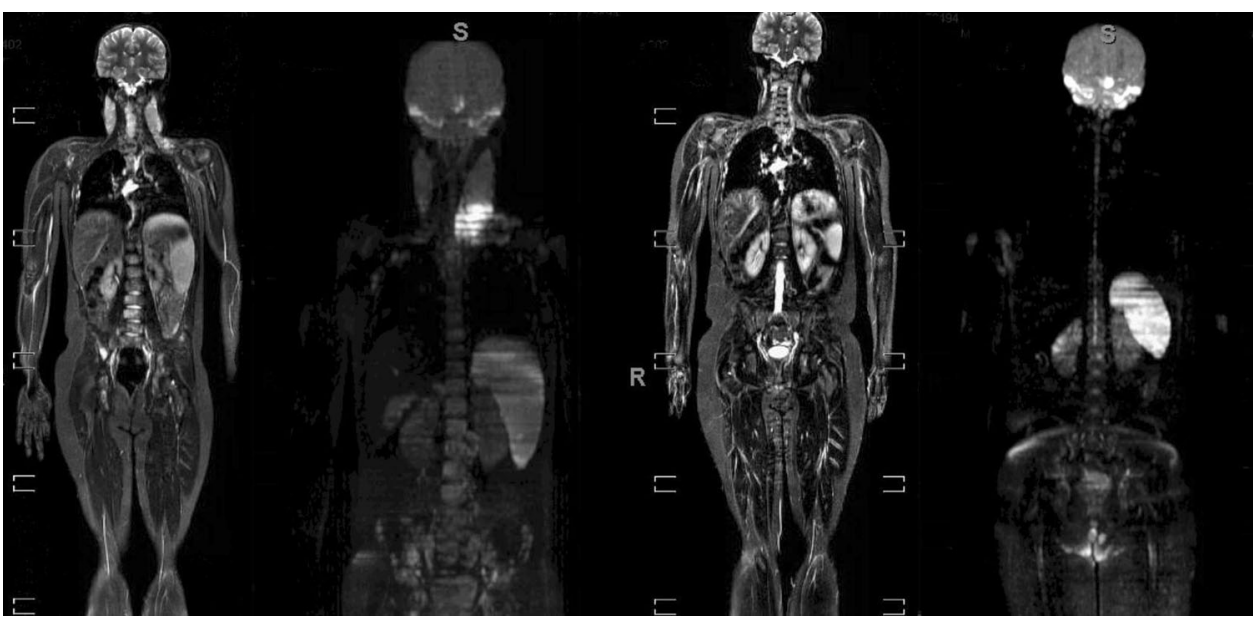

locations where MR scanners still of low availability, keeping in mind that a good interaction between the radiologists and radiologic technologists is needed to determine the ideal parameters for reading.

\section{Compliance with Ethics Guidelines}

Conflict of Interest Dr. Jose Luiz de Oliveira Schiavon and Dr. Jeannie Romani each declare no potential conflicts of interest. Dr. Henrique M. Lederman is a section editor for Current Radiology Reports.

Human and Animal Rights and Informed Consent This article does not contain any studies with human or animal subjects performed by any of the authors.

\section{References}

Papers of particular interest, published recently, have been highlighted as:

- Of importance;

•- Of major importance

1. Edwards AD, Arthurs OJ. Paediatric MRI under sedation: is it necessary? What is the evidence for the alternatives? Pediatr Radiol. 2011; 41(11):1353-64. doi:10.1007/s00247-011-2147-7.

Fig. 4 Sensible but less specific variant of WBMRI that includes only STIR images of a partial lower extremity on the search for osteonecrosis

A more sensible but less specific variant of WBMRI can be performed with only the coronal STIR sequence for some known pathologies, taking about $18 \mathrm{~min}$ for the whole body. Some of these pathologies include the search for osteonecrosis, which reduce the usual 30-min complete hip, knee, and ankle bilateral scan to a 9-min partial WBMRI STIR protocol (Fig 4).

On summary, most pediatric patients can be followed up using MRI-personalized protocols, avoiding the use of CT, and allowing more patients to be scanned hourly on
2. Dean DC 3rd, Dirks H, O’Muircheartaigh J, Walker L, Jerskey BA, Lehman K, Han M, Waskiewicz N, Deoni SC. Pediatric neuroimaging using magnetic resonance imaging during nonsedated sleep. Pediatr Radiol. 2014; 44(1):64-72. doi:10.1007/ s00247-013-2752-8.

3. Olsen ØE. MRI: how to perform a pediatric scan. Acta Radiol. 2013; 54(9):991-7. doi:10.1177/0284185112474917.

4. • Rozovsky K, Ventureyra EC, Miller E. Fast-brain MRI in children is quick, without sedation, and radiation-free, but beware of limitations. J Clin Neurosci. 2013; 20(3):400-5. doi:10.1016/j. jocn.2012.02.048. This article describes limitations of fast protocols over the brain

5. - Leite ED, Seber A, de Barbosa FG, Ginani VC, Carlesse FC, Gouvea RV, Zecchin VG, Carvalho CR, Szarf G, Lederman HM. Rapid, low-cost MR imaging protocol to document central nervous 
system and sinus abnormalities prior to pediatric hematopoietic stem cell transplantation. Pediatr Radiol. 2011; 41(6):749-56. doi:10.1007/s00247-011-1988-4. This article describes the use of fast protocols over sinus evaluation
6. Ashley WW Jr, McKinstry RC, Leonard JR, Smyth MD, Lee BC, Park TS. Use of rapid-sequence magnetic resonance imaging for evaluation of hydrocephalus in children. J Neurosurg. 2005;103(2 Suppl):124-30. 